\title{
Spectroscopic investigation of the interaction of bovine serum albumin with a novel cardiac agent V-09
}

\author{
Changyun Chen ${ }^{\mathrm{a}, *}$, Meihua Ma ${ }^{\mathrm{a}}$, Junqi Zhang ${ }^{\mathrm{b}}$, Lichen Wang ${ }^{\mathrm{b}}$ and Bingren Xiang ${ }^{\mathrm{b}}$ \\ ${ }^{a}$ Department of Chemistry, Nanjing Xiaozhuang University, Nanjing 210017, P. R. China \\ ${ }^{\mathrm{b}}$ Center for Instrumental Analysis, China Pharmaceutical University, Nanjing 210009, P. R. China
}

\begin{abstract}
This study employs fluorescence spectroscopy to characterize the binding properties of a newly synthesized cardiac agent, V-09, on bovine serum albumin (BSA). This compound shows the highest cardiac activity in the whole series. The binding constants $K$ at $25^{\circ} \mathrm{C}$ and $37^{\circ} \mathrm{C}$ are obtained, the values are $7.12 \times 10^{4} 1 \mathrm{~mol}^{-1}, 4.66 \times 10^{4} 1 \mathrm{~mol}^{-1}$, respectively. The standard enthalpy change $\left(\Delta H^{0}\right)$ and the standard entropy change $\left(\Delta S^{0}\right)$ are calculated to be $-27.13 \mathrm{KJ} \mathrm{mol}^{-1}$ and $1.854 \mathrm{~J} \mathrm{~mol}^{-1} \mathrm{~K}^{-1}$, which indicated that hydrophobic forces play major role in the interaction between $\mathrm{V}-09$ and BSA. The binding average distance between V-09 and BSA $(2.57 \mathrm{~nm})$ is obtained on the basis of the theory of Forster energy transfer.
\end{abstract}

Keywords: Bovine serum albumin, cardiac agent, interaction, fluorescence spectroscopy

\section{Introduction}

Bovine serum albumin (BSA), one of the major components in the plasma protein, is a single-chain 582 amino acid globular nonglycoprotein cross-linked with 17 cystine residues ( 8 disulfide bonds and 1 free thiol). It is divided into three linearly arranged, structurally distinct, and evolutionarily related domain (I-III); each domain is composed of two subdomains (A and B). BSA has two tryptophans, embedded in two different domains; Trp-134, located in proximity to the protein surface, but buried in hydrophobic pocket of domain I, and Trp-214, located in an internal part of domain II. Like other serum albumins, BSA has a wide range of physiological functions involving the binding, transport, and delivery of fatty acid, porphyrins, bilirubin, tryptophan, thyroxine, and steroids. It is home to specific binding sites for metals, pharmaceuticals and dyes [1-3]. The interaction between serum albumin and pharmaceutical agents is very important in the pharmacokinetics, pharmacodynamics and toxicology of pharmaceutical agents. The affinity of albumin for a particular drug determines the rate at which unbound or free drug becomes available to diffuse from the circulation to sites of action and metabolism. It is the unbound form of the drug that interacts with sites and exerts the agent's pharmacologic effect [4].

The great progress of today's biology has resulted in the better understanding on the pathological physiology and the treatment of congestive heart-failure (CHF). Neurocrine antagonist $\beta$-adrenergic receptor centagonist, the drug that can change myocardial rebuilding, has attracted more and more interests

\footnotetext{
* Corresponding author: C. Chen, Department of Chemistry, Nanjing Xiaozhuang University, Nanjing 210017, P. R. China. Tel.: +86 25 86569262; E-mail: yhjiangccy@126.com.
} 


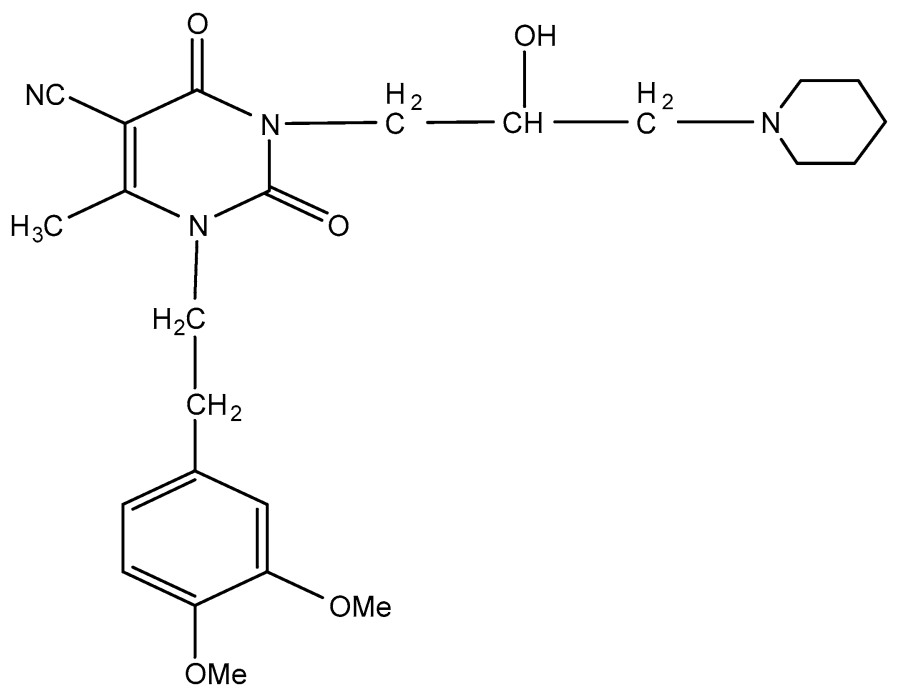

Fig. 1. The chemical structure of V-09.

for the treatment of CHF recently. This drug has the great potential to become the fourth type anti CHF medicine after the cardiotonic treatment, diuresis treatment, and blood vessel dilatation therapy. However, short period worsening of heart function was found in clinical testing with $\beta$-adrenergic receptor centagonist due to the side effect of this medicine.

To search for new anti-CHF medicine with high efficiency but low side effect, we designed and synthesized a series of anti-CHF drugs based on the principle of biological equivalent electron arrange and structural combination. These drugs are the combination of two parts, one part with the 1-[2-(3,4-dimethoxy)phenylethyl]-5-cyano-6-methyl-uracil that normally shows cardiotonic activity, while another part with the side chain of 2 -amino propanol which usually exists in $\beta$-adrenergic receptor centagonist. We found V-09 (structure shown in Fig. 1) has the highest cardiotonic activity in the whole series [5].

In the present investigation, we employ fluorescence spectroscope to characterize the binding properties of V-09 on BSA. The results of the study provide a molecular basis for elucidating the mechanism of drug action and predicting unfavorable drug interaction.

\section{Materials and methods}

\subsection{Materials and instruments}

Bovine serum albumin (BSA) was purchased from Beijing Huamei Biotechnology Company (China). All BSA solution was prepared in $\mathrm{pH} 7.40$ buffer solution and BSA stock solution was kept in the dark at $4^{\circ} \mathrm{C}$. V-09 was synthesized by China Pharmaceutical University. It was characterized by IR, ESI-MS: $457(\mathrm{M}+\mathrm{H}),{ }^{1} \mathrm{HNMR}: 1.46-1.58(2 \mathrm{H}), 1.60-2.63(4 \mathrm{H}), 2.42-2.64(6 \mathrm{H}), 2.32(3 \mathrm{H})$, $2.95(2 \mathrm{H}), 3.88(6 \mathrm{H}), 3.92-4.08(2 \mathrm{H}), 4.12(2 \mathrm{H}), 4.12-4.21(1 \mathrm{H}), 6.69-6.84(3 \mathrm{H})$ and element analysis: $\mathrm{C}_{24} \mathrm{H}_{32} \mathrm{~N}_{4} \mathrm{O}_{5}, \mathrm{C}: 63.53, \mathrm{H}: 7.05, \mathrm{~N}: 12.21$ (Req. 63.14, 7.07, 12.27). $\mathrm{NaCl}$ (analytical grade, 0.9\%) solution was used to maintain the ion strength. Buffer $(\mathrm{pH} 7.40)$ consist of Tris $\left(0.2 \mathrm{~mol} \mathrm{l}^{-1}\right)$ and 
$\mathrm{HCl}\left(0.1 \mathrm{~mol} \mathrm{l}^{-1}\right)$, and the $\mathrm{pH}$ was adjusted to 7.40 by adding $0.5 \mathrm{~mol}^{-1} \mathrm{NaOH}$ when the experiment temperature was higher than $298 \mathrm{~K}$. The $\mathrm{pH}$ was checked with a suitably standardized meter.

The absorption spectra were recorded on a TU-1901 spectrophotometer (Beijing) and the fluorescence spectra were recorded on a Perkin Elmer LS-50B spectrofluorophotometer (USA), using $5 \mathrm{~nm} / 5 \mathrm{~nm}$ slit widths.

\subsection{Experimental methods}

BSA solution was transferred into a $10 \mathrm{ml}$ volumetric flask, then $0.9 \% \mathrm{NaCl}$ solution, buffer ( $\mathrm{pH} 7.40$ ) and V-09 operating solution was added in different amounts to the flask. The mixture was diluted to a certain volume with doubly distilled water, and mixed thoroughly. After 10 min the samples were excited at $278 \mathrm{~nm}$ and the fluorescence intensity was monitored at $300-500 \mathrm{~nm}$.

\section{Results and discussion}

\subsection{Binding constants of BSA with V-09}

The fluorescence intensity $(F)$ of BSA was decreased regularly with increasing concentration of V-09, and nearly no shift was observed for the emission wavelengths if the concentration of BSA was fixed. The fluorescence quenching spectra of BSA with different concentrations of V-09 at different temperatures $\left(25^{\circ} \mathrm{C}, 37^{\circ} \mathrm{C}\right)$ are shown in Figs 2 and 3, respectively.

The static quenching and dynamic quenching were differentiated by the results at different temperatures. The quenching rate constants decrease with increased temperature for the static quenching, but the reversed effect was observed for the dynamic quenching [6]. The possible quenching mechanism can be interpreted by the fluorescence quenching spectra of the proteins and the $F_{0} / F-C$ (Stern-Volmer) curves of BSA with V-09 at different temperatures $\left(25^{\circ} \mathrm{C}, 37^{\circ} \mathrm{C}\right)$ as shown in Fig. 4.

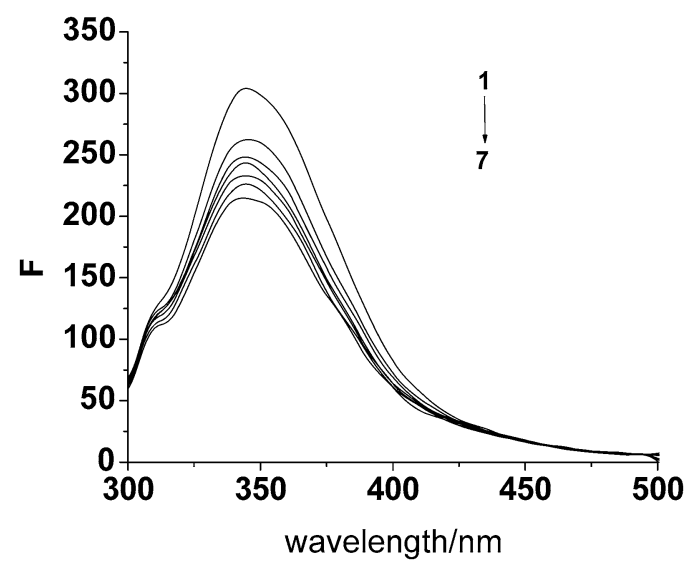

Fig. 2. Fluorescence emission spectra of BSA $\left(5.0 \times 10^{-7} \mathrm{~mol}^{-1}\right)$ at $25^{\circ} \mathrm{C}$ in the absence and presence of increasing amount of V-09 $\left(5.0 \times 10^{-7} \mathrm{~mol} \mathrm{l}^{-1}\right)$ from 1 to $7: 0,1.0,2.0,3.0,4.0,5.0,6.0$. 


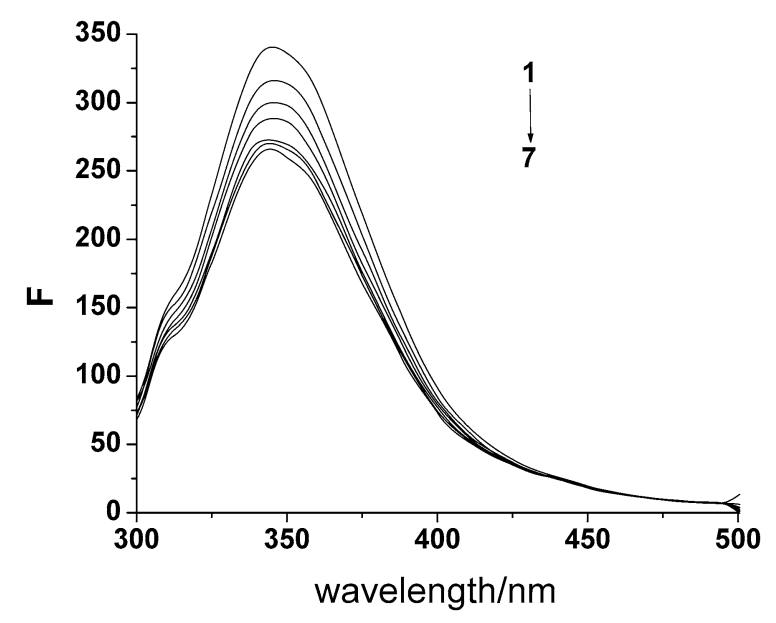

Fig. 3. Fluorescence emission spectra of BSA $\left(5.0 \times 10^{-7} \mathrm{~mol} \mathrm{l}^{-1}\right)$ at $37^{\circ} \mathrm{C}$ in the absence and presence of increasing amount of $\mathrm{V}-09\left(5.0 \times 10^{-7} \mathrm{~mol} \mathrm{l}^{-1}\right)$ from 1 to $7: 0,1.0,2.0,3.0,4.0,5.0,6.0$.

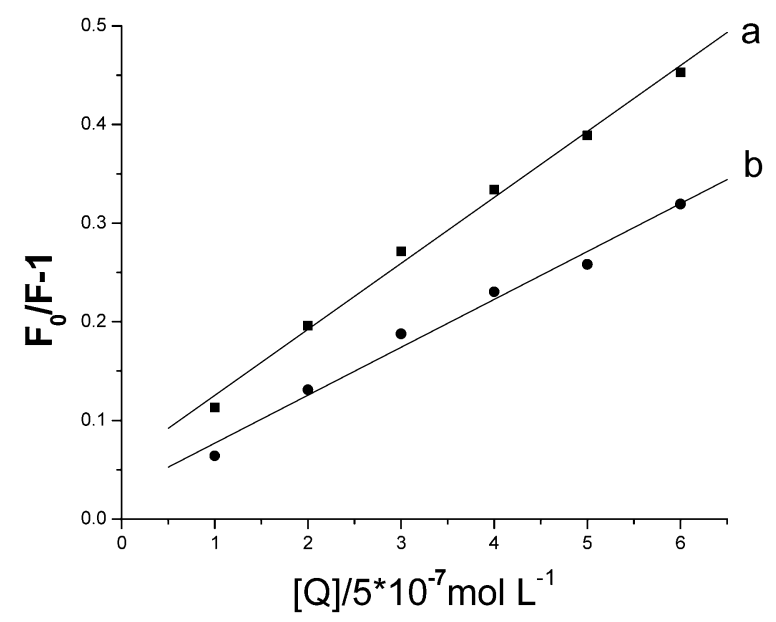

Fig. 4. The Stern-Volmer curve of BSA $\left(5.0 \times 10^{-7} \mathrm{~mol}^{-1}\right)$ quenched by V-09, (a) $25^{\circ} \mathrm{C}$; (b) $37^{\circ} \mathrm{C}$.

It can be found from Fig. 4 that the Stern-Volmer plots are linear and the slopes decrease with increasing temperature. This indicates the static quenching interaction between V-09 and BSA. In order to confirm this point, the procedure was assumed to be dynamic quenching. The quenching is presented by

$$
F_{0} / F=1+K_{\mathrm{q}} \tau_{0}[Q]=1+K_{\mathrm{sv}}[Q]
$$

where $F$ and $F_{0}$ are the fluorescence intensity with and without quencher, respectively. $K_{\mathrm{q}}$ is quenching rate constant of the biomolecule, $K_{\mathrm{sv}}$ is the Stern-Volmer quenching constant, $\tau_{0}$ is the average lifetime of the biomolecule without quenching, and $[Q]$ is the concentration of quenching. Obviously,

$$
K_{\mathrm{sv}}=K_{\mathrm{q}} \tau_{0}
$$


Table 1

The dynamic quenching and binding constants between V-09 and BSA

\begin{tabular}{lccc}
\hline$T\left({ }^{\circ} \mathrm{C}\right)$ & $K_{\mathrm{q}}\left(1 \mathrm{~mol}^{-1} \mathrm{~s}^{-1}\right)$ & $R$ & $K\left(1 \mathrm{~mol}^{-1}\right)$ \\
\hline 25 & $7.12 \times 10^{12}$ & 0.9941 & $7.12 \times 10^{4}$ \\
37 & $4.66 \times 10^{12}$ & 0.9999 & $4.66 \times 10^{4}$ \\
\hline
\end{tabular}

Because the fluorescence lifetime of the biopolymer is $10^{-8} \mathrm{~s}^{-1}$ [7], $K_{\mathrm{sv}}$ is the slope of linear regressions of Fig. 4. According to Eq. (2), the quenching constant $K_{\mathrm{q}}$ can be obtained and are listed in Table 1 together with the correlation coefficients. However, the maximum scatter collision quenching constant $K_{\mathrm{q}}$ of various quenchers with the biopolymer is $2 \times 10^{10} 1 \mathrm{~mol}^{-1} \mathrm{~s}^{-1}$. Obviously, the rate constant of protein quenching procedure initiated by $\mathrm{V}-09$ is greater than the $K_{\mathrm{q}}$ of the scatter procedure. This means that the quenching is not initiated by dynamic collision but from the formation of a complex. The $K_{\mathrm{sv}}$ obtained from Stern-Volmer plot is the binding constant $K$ for static quenching. The value of binding constant is also given in Table 1.

It is shown that the binding between $\mathrm{V}-09$ and BSA is remarkable and the effect of temperature is small. Thus, V-09 can be stored and removed by the proteins in the body.

\subsection{Determination of the acting force}

The action force between a drug and a biomolecule may include hydrogen bond, van der Waals force, electrostatic force and hydrogen bond interaction force and so on. Because the temperature effect is very small, the interaction enthalpy change can be regarded as a constant if the temperature range is not too wide. Therefore, from the following equations,

$$
\begin{aligned}
& \ln \left(K_{2} / K_{1}\right)=\Delta H\left(1 / T_{1}-1 / T_{2}\right) / R, \\
& \Delta G=\Delta H-T \Delta S=-R T \ln K,
\end{aligned}
$$

where $\Delta H, \Delta G$ and $\Delta S$ are, respectively, enthalpy change, free energy change, $\Delta H$ and $\Delta S$ for the binding interaction between V-09 and BSA are calculated to be $-27.13 \mathrm{~kJ} \mathrm{~mol}^{-1}$ and $1.854 \mathrm{~J} \mathrm{~mol}^{-1} \mathrm{~K}^{-1}$. In other words, enthalpy change $\Delta H<0$ and entropy change $\Delta S>0$. So it can be deduced that the acting force are mainly a hydrophobic interaction [8].

\subsection{The binding distance between V-09 and BSA}

According to the Förster's theory [9], the efficiency of energy transfer, $E$, is given by:

$$
E=R_{0}^{6} /\left(R_{0}^{6}+r^{6}\right),
$$

where $r$ is the donor-acceptor distance and $R_{0}$ is the distance at $50 \%$ transfer efficiency.

$$
R_{0}^{6}=8.8 \times 10^{-25} K^{2} n^{-4} \phi J
$$

where $K^{2}$ is the orientation factor between the emission dipole of the donor and the absorption dipole of the acceptor. The dipole orientation factor, $K^{2}$, is the least certain parameter in calculation of the critical transfer distance, $R_{0}$. Although theoretically $K^{2}$ can range from 0 to 4 , the extreme values require 


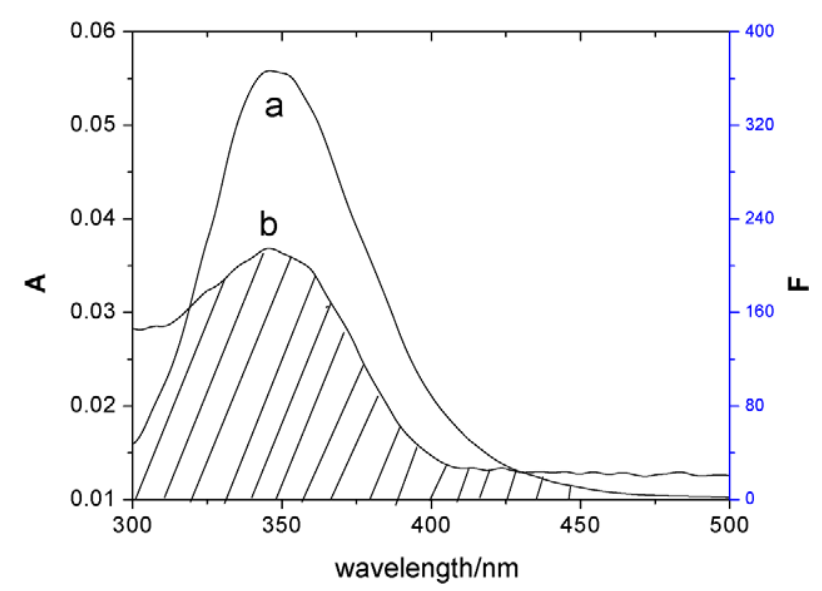

Fig. 5. The overlap of the fluorescence emission spectra of BSA (a) and the absorption spectra of V-09 (b) $\mathrm{c}(\mathrm{V}-09)=$ $5.0 \times 10^{-7} \mathrm{~mol} / \mathrm{l} ; \mathrm{c}(\mathrm{BSA})=5.0 \times 10^{-7} \mathrm{~mol} / \mathrm{l} ; \lambda_{\mathrm{ex}}=278 \mathrm{~nm}$.

very rigid orientations. If both the donor and acceptor are tumbling rapidly and are free to assume any orientation, then $K^{2}$ equals $2 / 3$ [10]. If only the donor is free to rotate, then $K^{2}$ can vary from $1 / 3$ to $4 / 3$ [11]. The validity of assuming a value of $2 / 3$ has been discussed often [12-14]. Therefore, $K^{2}=2 / 3$ was used for the dynamic averaging of relative donor-acceptor positions in the V-09-BSA system; $n(=1.4)$ is the refractive index of medium [15], $\phi$ is the fluorescence quantum yield of the donor (its value was taken as 0.10 [16]) and $J$ is the overlap integral of the fluorescence emission spectra of the donor and the absorption spectra of the acceptor. $J$ is given by:

$$
J=\left[\sum F(\lambda) \varepsilon(\lambda) \lambda^{4} \Delta \lambda\right] /\left[\sum F(\lambda) \Delta \lambda\right]
$$

where $F(\lambda)$ is the fluorescence intensity of fluorescence reagent when the wavelength is $\lambda . \varepsilon(\lambda)$ is the molar absorbance coefficient at the wavelength of $\lambda$. From these relationships, $J, E$ and $R_{0}$ can be calculated; so the value of $r$ also can be calculated.

Figure 5 is the overlap of the fluorescence spectra of BSA and the absorption spectra of V-09 when the molar ratio is $1: 1$.

From Fig. 5, the overlap integral calculated according to above relationship is $2.98 \times 10^{-15} \mathrm{~cm}^{3} 1 \mathrm{~mol}^{-1}$. So the value of $R_{0}$ is $1.85 \mathrm{~nm}$, and the value of $r$ is $2.57 \mathrm{~nm}$. This result, which corresponds to the theory of energy transfer [17], indicates that the binding reaction of V-09 to BSA is through energy transfer, which will quench the fluorescence of the protein. For BSA, it has two tryptophan residues: Trp-214 is located in a hydrophobic fold and the additional tryptophan (Trp-135) is located on the surface of molecule [18,19]. In this paper, V-09 is probably bound to Trp-214 residue mainly through the hydrophobic interaction according to the thermodynamic results. However, interaction between the Trp-135 and V-09 cannot be precluded, so the distance calculated here is actually the average value between the bound V-09 and two tryptophan residues in BSA.

\subsection{Synchronous fluorescence studies}

The fluorescence of BSA comes from tyrosine, tryptophan and phenylalanine residues. According to Miller [20], with large $\Delta \lambda$ values such as $60 \mathrm{~nm}$, the synchronous fluorescence of BSA is characteristic 


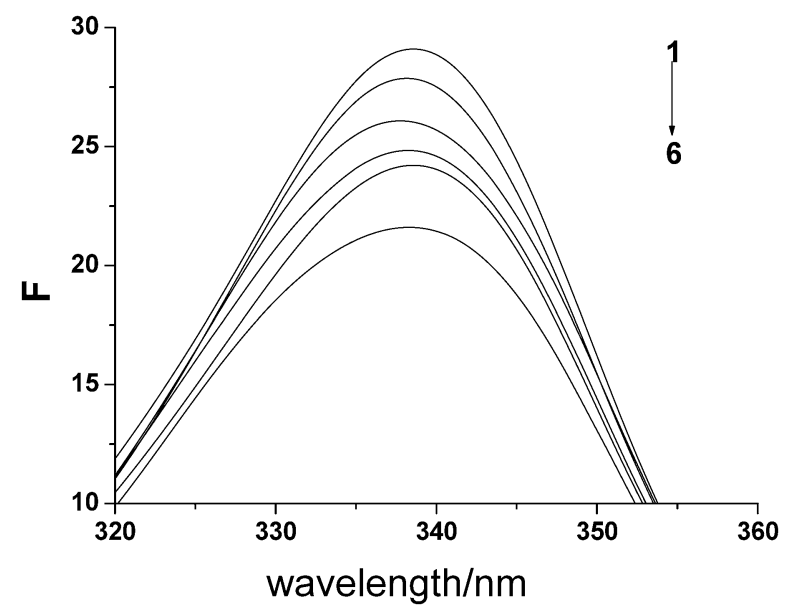

Fig. 6. Synchronous fluorescence spectra of BSA $\left(5.0 \times 10^{-7} \mathrm{~mol} \mathrm{l}^{-1}\right)$ with $\Delta \lambda=60 \mathrm{~nm}$ in the absence and presence of increasing amount of V-09 $\left(5.0 \times 10^{-7} \mathrm{~mol} \mathrm{l}^{-1}\right)$ from 1 to $6: 0,1.0,2.0,3.0,4.0,5.0$.

of tryptophan residue. Figure 6 shows the effect of addition of V-09 on the synchronous fluorescence spectra of BSA when $\Delta \lambda=60 \mathrm{~nm}$.

It is indicated that addition of V-09 results in the strong fluorescence quenching of tryptophan and nearly no wavelength shift is observed when the concentration of V-09 increased from 0 to $50 \mu \mathrm{M}$. This reflects the microenvironment of tryptophan residue is almost not affected by the drug binding.

\section{Conclusions}

In summary, the experimental results indicate that V-09 can bind on the surface of BSA strongly, which is driven mainly by hydrophobic interactions, and cause the fluorescence of BSA to be quenched. In addition, based on the above discussions, V-09 has nearly no effect on the serum protein conformation.

\section{References}

[1] J.Q. Liu, J.N. Tian, W.Y. He et al., In vitro study on the binding of anti-coagulant vitamin to bovine serum albumin and the influence of toxic ions and common ions on binding, J. Pharm. Biomed. Anal. 35 (2004), 671-677.

[2] D.C. Carter and J.X. Ho, Structure of serum albumin, Adv. Protein Chem. 45 (1994), 153-203.

[3] T. Peters, All About Albumin: Biochemistry, Genetics and Medicals Applications, Academic Press, San Diego, CA, 1995.

[4] W.Y. Zhong, Y.C. Wang, J.S. Yu et al., The interaction of human serum albumin with a novel antidiabetic agent - SU-118, J. Pharm. Sci. 93 (2004), 1039-1046.

[5] J.Q. Zhang, L.C. Wang, Z.Z. Jiang and M. Lin, Synthesis and biologic activities on 1-\{1-[2-(3,4-dimethoxy)phenylethyl]5-cyano-6-methyl-uracil-3yl\}-3-substituted amino-2-propanol compounds, J. Chin. Pharm. Univ. 34 (2003), 396-399.

[6] G.Z. Chen, X.Z. Huang, J.G. Xu, Z.Z. Zheng and Z.B. Wang, The Methods of Fluorescence Analysis, 2nd edn, Science Press, Xi' an, 1991.

[7] J.R. Lakowica and G. Weber, Quenching of fluorescence by oxygen: A probe for structural fluctuations in macromolecules, Biochemistry 12 (1973), 4161-4170.

[8] P.D. Ross and S. Subramanian, Thermodynamics of protein association reaction: forces contribution to stability, Biochemistry 20 (1981), 3096-3102.

[9] T. Főrster, Modern Quantum Chemistry, Vol. 3, O. Sinanoglu, ed., Academic Press, New York, 1996.

[10] T. Förster, Transfer mechanisms of electronic excitation, Discuss Faraday Soc. 27 (1959), 7-10.

[11] C.W. Wu and L. Stryer, Proximity relationships in rhodopsin, Proc. Natl. Acad. Sci. USA 69 (1972), 1104-1108. 
[12] J.R. Lakowica, Principles of Fluorescence Spectroscopy, 2nd edn, Kluwer/Plenum, New York, 1999.

[13] Z. Hillel and C.W. Wu, Statistical interpretation of fluorescence energy transfer measurements in macromolecular systems, Biochemistry 15 (1976), 2105-2113.

[14] L. Stryer, Fluorescence energy transfer as a spectroscopic ruler, Annu. Rev. Biochem. 47 (1978), 819-846.

[15] S. Kasai, T. Horie, T. Mizuma and S. Awazu, Fluorescence energy transfer study of the relationship between the lone tryptophan residue and drug binding sites in human serum albumin, J. Pharm. Sci. 76 (1987), 387-392.

[16] E.B. Charies and B.S. Hudson, Spectroscopic studies of binding and proximity relationships for fatty acids and bilirubin, J. Biol. Chem. 254 (1979), 391-400.

[17] W. Marty, M. Boiret and M. Deumin, How to illustrate ligand-protein binding in a class experiment: An elementary fluorescent assay, J. Chem. Educ. 63 (1986), 365-366.

[18] B.F. Peterman and K.J. Laidler, Study of reactivity of tryptophan residues in serum albumins and lysozyme by Nbromosuccinamide fluorescence quenching, Arch. Biochem. Biophys. 199 (1980), 158-164.

[19] M.V. Pierre, V.D. Tuan, A.C. Ribou, V. Jean and J.M. Salmon, Native fluorescence and mag-indo-1-protein interaction as tools for probing unfolding and refolding sequences of the bovine serum albumin subdomain in the presence of guanidine hydrochloride, J. Protein Chem. 19 (2000), 431-439.

[20] J.N. Miller, Recent advances in molecular luminescence analysis, Proc. Anal. Div. Chem. Soc. 16 (1979), $203-208$. 


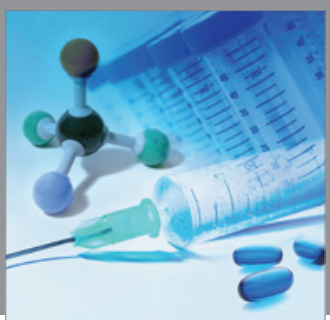

International Journal of

Medicinal Chemistry

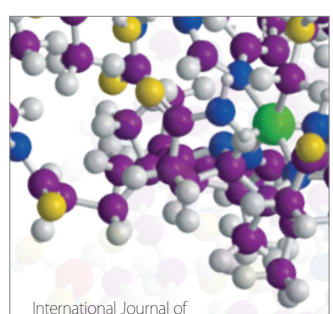

Carbohydrate Chemistry

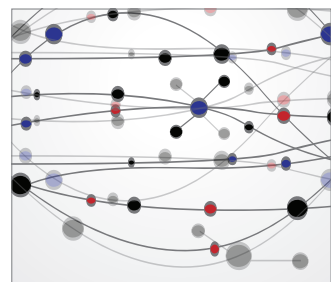

The Scientific World Journal
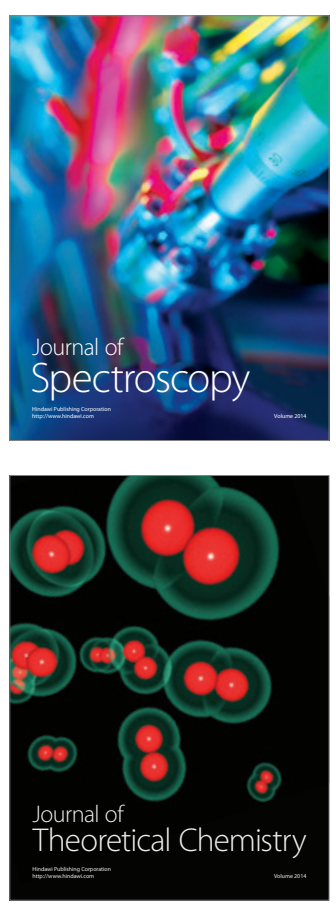
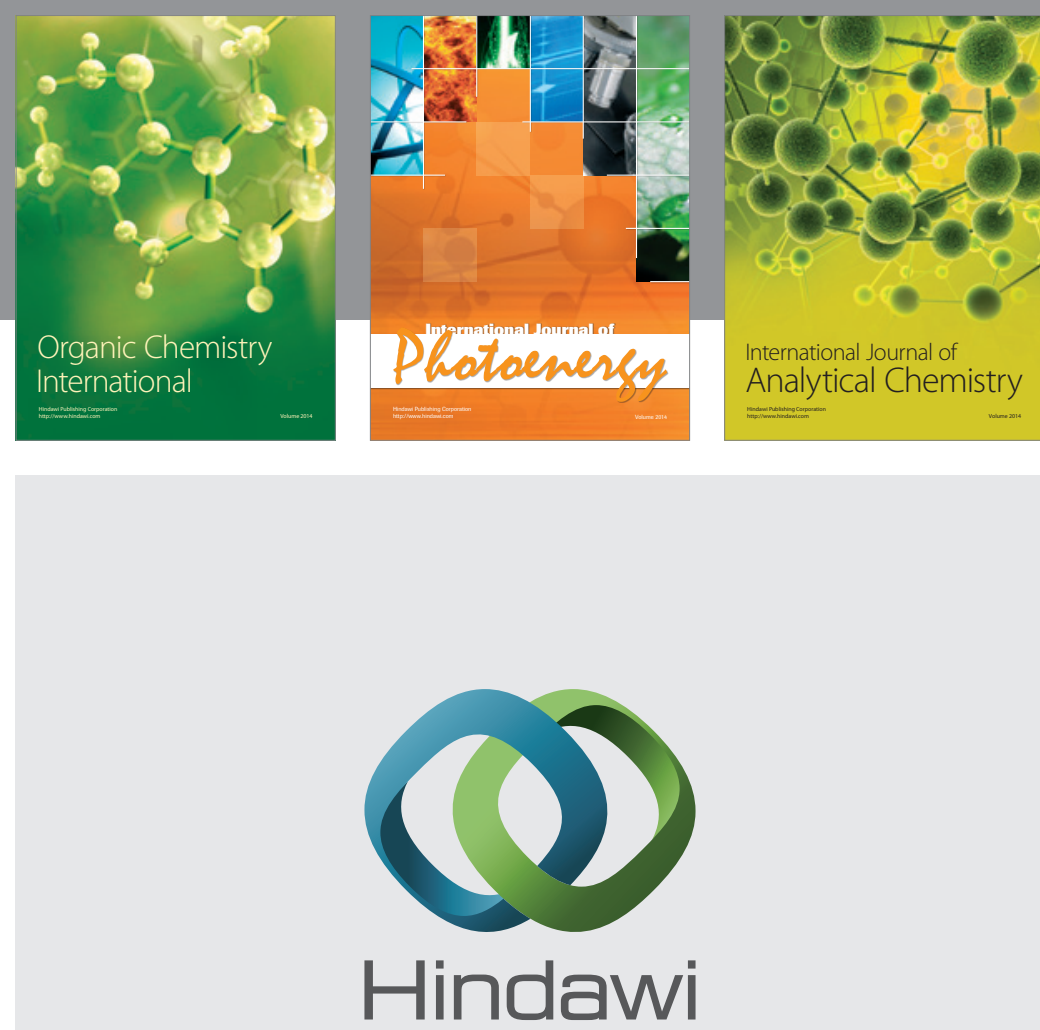

Submit your manuscripts at

http://www.hindawi.com
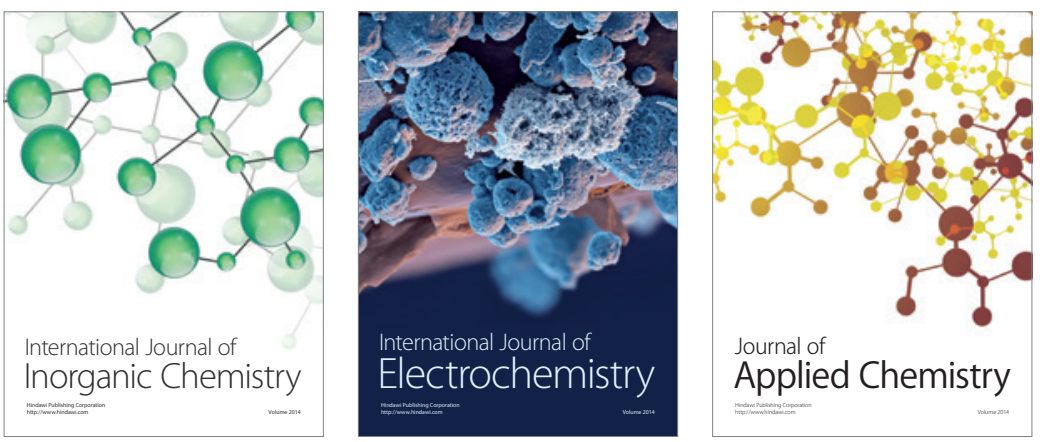

Journal of

Applied Chemistry
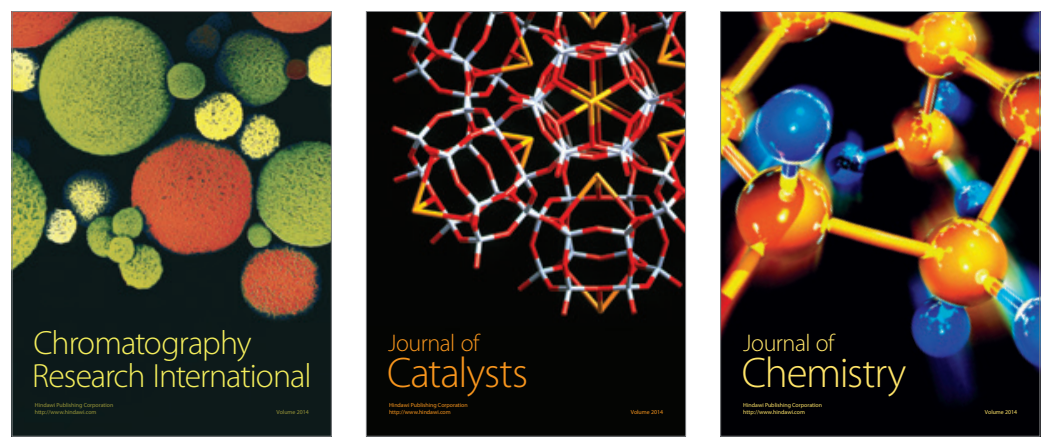
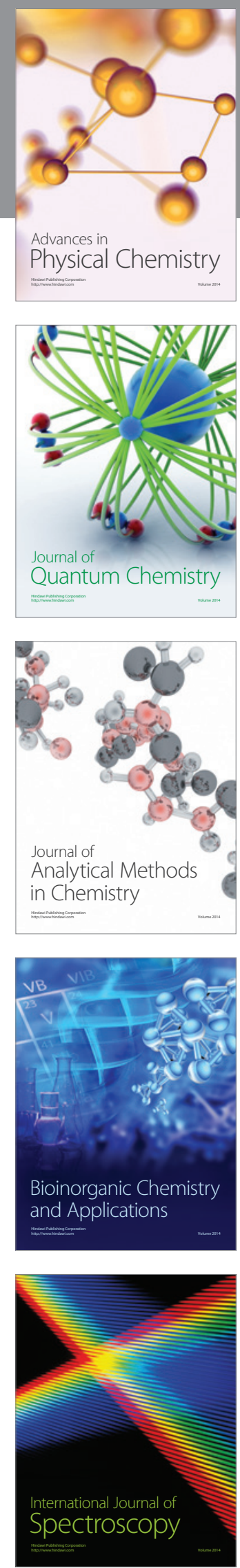